\title{
On the Azimuthally Anisotropy Effects of Polarization for Altimetric Measurements
}

\author{
Frédérique Rémy, Benoît Legrésy, and Jérôme Benveniste
}

\begin{abstract}
We have investigated the effect of the radar-altimeter antenna polarization on European Remote-sensing Satellite and Envisat observations of the media penetrable by a radar microwave such as ice sheets. This effect is due to the complex interaction between the radar wave, the subsurface backscatter, and the antenna polarization direction. It is modulated by the angle between the antenna polarization and the direction of the anisotropy of the target. Thus, it depends on both the anisotropy direction and the interaction between the radar wave and the reflecting surface. This effect leads to one of the most complex and least understood errors of radar altimetry over ice sheets and can be clearly identified when looking at the crossover differences between ascending and descending satellite tracks. The crossover differences are as large as a few decibels for a backscattering coefficient and a few meters for height, and affect more strongly the Ku-band than the S-band. This causes limitations and difficulties for the processing of altimetric observations, for instance when comparing time series from different satellites whose polarization geometry differs. This will be the case when a new altimeter will fly on a different orbit, as planned for CryoSat. Nevertheless, the ability of both the roughness anisotropy direction and the subsurface modulation to be inverted with satisfactory precision by using simultaneous observations at crossover points between two different satellites is demonstrated here. Thus, it offers a unique way of describing this error accurately to correct for it.
\end{abstract}

Index Terms-Altimetry, Antarctica, ice sheet.

\section{INTRODUCTION}

$\mathbf{O}$ NE objective of satellite radar altimetry above an ice sheet is to provide a homogeneous time series of height measurements to determine whether it is growing or shrinking. Another goal is to derive snowpack characteristics, such as internal stratification or surface roughness, which is related to the climate. To determine both elevation and climatic parameters, the altimetric measurement should be acquired and processed carefully by detecting, describing, and understanding all sources or errors and applying the derived error corrections.

Among all the radar-altimetry errors above the ice sheets, the most mysterious and least understood is probably the way the altimeter perceives the surface or the snowpack anisotropy. This effect, which was first pointed out and explained by Legrésy et al. [6], can be detected at the crossover points, owing to the different flight trajectories azimuth, where measurements from ascending and descending tracks of European Remote-

Manuscript received May 20, 2005; revised April 30, 2006.

F. Rémy and B. Legrésy are with the Laboratoire d'Etudes en Géophysique et Océanographie Spatiales (LEGOS), Centre National de la Recherche Scientifique (CNRS-CNES-IRD-UPS), 31401 Toulouse Cedex 9, France (e-mail: Frederique.remy@cnes.fr).

J. Benveniste is with the European Space Agency-ESRIN, 00044 Frascati Rome, Italy.

Digital Object Identifier 10.1109/TGRS.2006.878444 sensing Satellite 1 (ERS-1) radar altimetry show a systematic difference as large as a few meters for the height and a few decibels for the backscattering coefficient. The same effect is observed for the Envisat altimeter whose characteristics are the same as the ERS altimeters [see Fig. 1(a) and (b)]. The effect has been explained as being due to the interaction between the linearly polarized wave of the ERS altimeter and the backscattering medium anisotropy. This anisotropy is caused either by a surface with a "prevailing sculpted direction" [6] or by a snowpack with an anisotropic e-folding, e.g., a snowpack with an extinction path length that depends upon the azimuthal orientation of the polarization vector [1] (see Fig. 2 for a simplified illustration). Altimetric observations from previous satellites such as Seasat or Geosat with a circular polarization antenna appear not to be affected.

This induced effect of anisotropy is very difficult to correct in altimetric data. First, it depends on the sensitivity to the subsurface backscatter, which has not yet been characterized. Second, it depends on the direction of the wind-driven features or more generally on the direction of the roughness anisotropy. This direction can only be estimated from scatterometer data [8], [11]. But since the scatterometer is a slant-looking radar, it does not detect the surface roughness at the same scale and at the same snow depth as the nadir-looking radar altimeter. A detailed study of the azimuthal anisotropy, as observed by different active or passive microwave slant-angle sensors, can be found in [8], but very little is known on this type of effect for a nadir-looking sensor.

The error induced by this anisotropy has a several consequences that limit or complicate the straightforward use of the altimetry data acquired over the ice sheets.

1) It precludes the use of a crossover analysis, i.e., comparing the data from ascending and descending tracks at crossover points in order to correct for systematic errors above the ice sheets as it is usually done elsewhere. A systematic difference between ascending and descending tracks should first be removed [12]. But this correction adds uncertainties and bias. Indeed, it is known that seasonal and interannual shifts in the wind direction alter the interface orientation and crystal shapes [2].

2) Long time-series data become more difficult to interpret in terms of volume variations. In fact, nothing ensures that the katabatic wind, and thus the wind-induced surface or snowpack characteristics remain stable over time. In case of a climate change, one can expect changes in atmospheric patterns, such as temperature inversion, and hence in the amplitude and direction of wind-induced features. Legrésy et al. [6] have shown that a $5^{\circ}$ change in the direction of the wind-driven features results in 

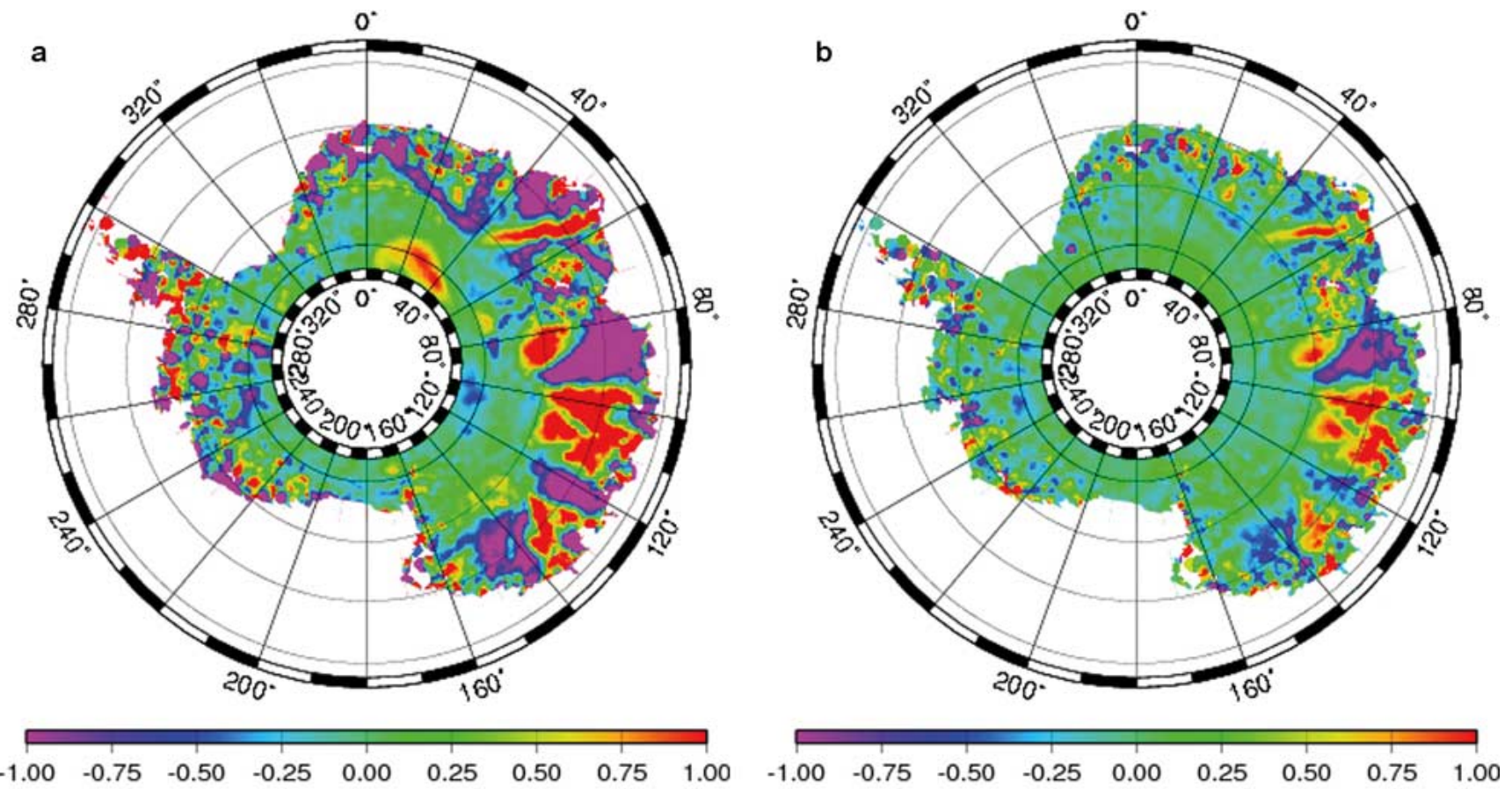

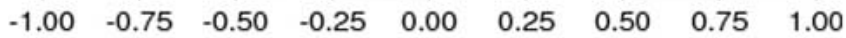

Ku-band backscattering difference (dB)

Ku-band leading edge difference $(\mathrm{m})$
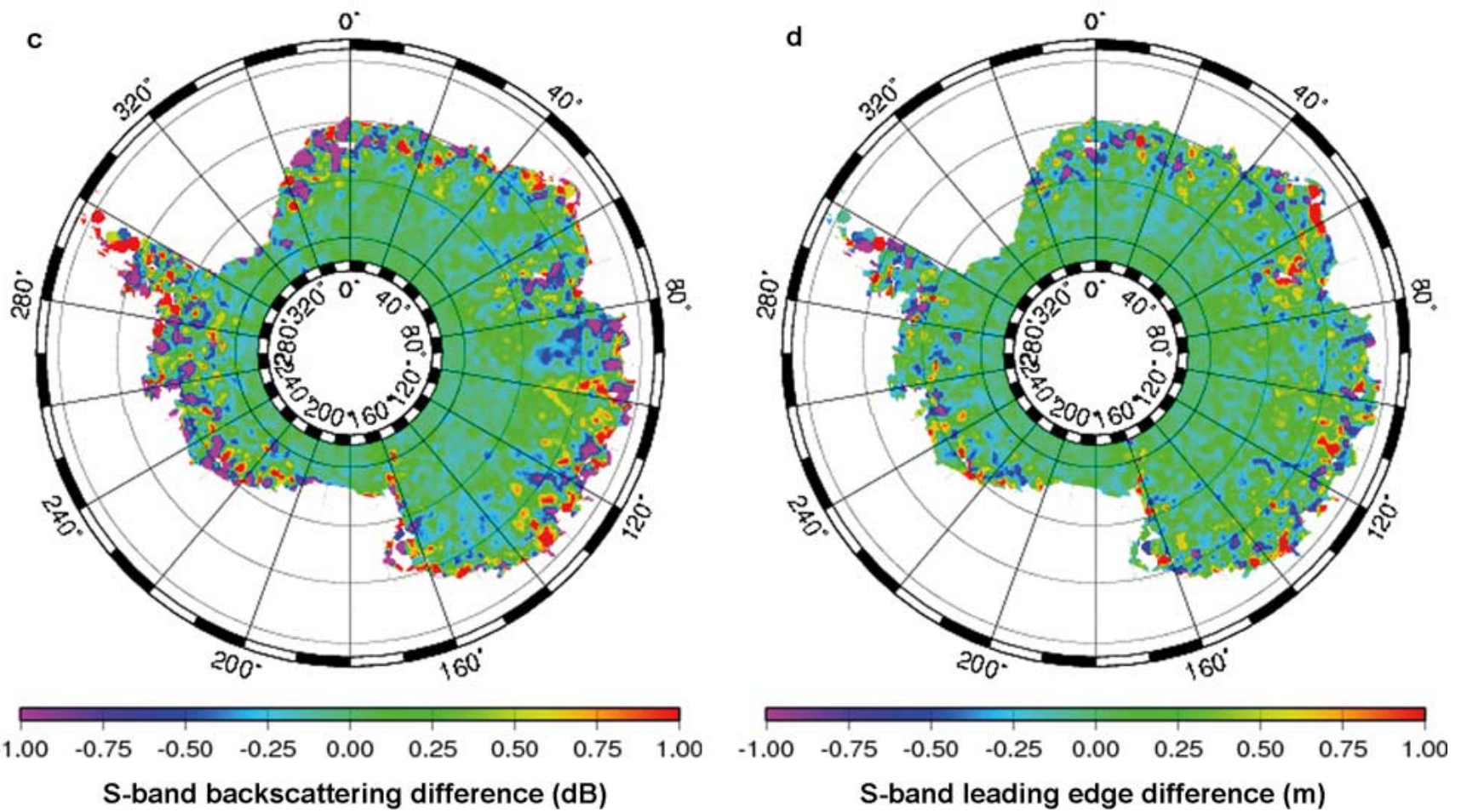

Fig. 1. Difference between ascending and descending tracks of the Envisat radar altimeter for the cycle 26. (a) For backscattering coefficient in Ku-band (expressed in decibels). (b) For the leading edge and thus the height for Ku-band (expressed in meters). (c) and (d) are the same as (a) and (b) for the S-band, respectively. Note that the intensity of the difference is very high near the coast with a distinct 100-km scale pattern, and that it decreases with latitude.

a 10-cm change in elevation for a classical Antarctica condition.

3) As we will see, this error depends on the radar frequency, such that it complicates the interpretation of observations made by altimeters operating at different frequencies. Legrésy et al. [7] or Papa et al. [9] have also recently shown the important contribution to continental studies of a dual-frequency altimeter, such as the one onboard Envisat.

4) This error greatly complicates the comparison of two radar altimeters with either different orbit inclinations or different antenna polarizations. The ERS and Envisat data series cannot be easily compared with the data from the previous altimeters, Seasat and Geosat whose 


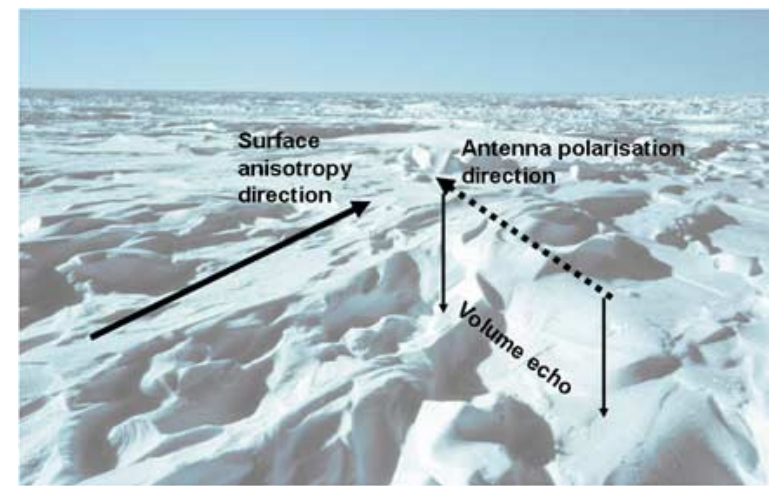

Fig. 2. Sastrugi field suggesting the wind effect on the surface and snowpack and a schematic explanation. These sastrugi are wind-driven erosion features, and the direction of the field follows the prevailing wind direction. Depending on the geometry between the antenna polarization and the sastrugi-field directions, either the interaction with the snowpack is more or less intense [6], or the internal extinction is more or less intense [1].

antennas were circularly polarized. It will not be possible to compare ERS and Envisat data series with the data from any future altimeters either in a different orbit or with a different antenna polarization direction, as it is the case for CryoSat-2.

Conversely, another question is whether a comparison of the data from two different satellites with different antenna polarization directions would yield a better description of the roughness anisotropy and of the induced error in the altimetric observations. It can be demonstrated that by using two linearly polarized altimeters on different orbits, it is possible to estimate the small-scale surface or snowpack anisotropy as well as the wind direction, and then correct for the error. We will demonstrate that a relation exists between the final precision of these parameters and the chosen spatial scale.

It should also be noticed that this error affects all natural or human-made surfaces exhibiting a prevailing surface direction, and that the methodology explained here can be applied elsewhere, for instance above deserts. Note that such an effect may also slightly affect the altimetry data acquired over an ocean, the modulation, if any, being more likely to be due to a microroughness and electromagnetic bias. This paper will focus on the Antarctica ice sheet, namely, on a surface where the modulation is due to the subsurface echo.

Initially, this study took place in the context of the launch of CryoSat by the European Space Agency (ESA). Unfortunately, the satellite was lost in October 2005 due to a launch failure. Nevertheless, the ESA will rebuild the satellite for a new launch in 2009. Furthermore, there will be future altimeters with different antenna or orbital characteristics, which will fly above the ice sheets. For the purpose of this paper, we focus on the future launch of CryoSat-2, but this study can still be applied to many other satellites.

\section{EFFECT OF ANTENNA Polarization AND ANISOTROPY ON RADAR MEASUREMENTS}

\section{A. Possible Causes}

The systematic differences between the data acquired at crossover points are a very persistent characteristic of ERS-1, ERS-2, and Envisat radar altimetry [Fig. 1(a) and (b)]. This ar- tifact has been observed for all kinds of orbits of ERS-1 (the 3-, 35- and 168-day repeat orbits) over the Antarctic ice sheet and the Greenland ice sheet, but not over the ocean, at least not with the same amplitude. These differences have been observed for elevation, for backscattering coefficient, and for all waveformshape parameters [1], [6]. When the backscattering coefficient is found to increase, other waveform parameters (such as leading edge width and trailing edge) are also found to increase, and the surface height is found to decrease proportionally [compare Fig. 1(a) with (b)]. This relation indicates that the crossover differences are due to a different sensitivity to the subsurface echo, which is related to a geometric orientation of the radar footprint with respect to the ground surface. Two explanations of this phenomenon have been previously suggested, as follows.

The first one, which is given by Legrésy et al. [6], is based upon the specific surface features of the ice sheet, namely the elongated wind-driven structures (Fig. 2). These may include erosion, snow drift or snow deposition figures due to the persistent and strong katabatic winds. These features are found on various scales, from the centimeter scale (microroughness) to the sastrugi scale (1-m scale), up to the megadune scale (100-m scale), [4], [10]. The sastrugi are so elongated along the wind direction that they can be used to estimate the wind direction either by in situ observations [3] or by remote-sensing observations [11]. Azimuth modulation of slant-looking microwave sensors is now thoroughly documented and well known (see for example, [5]).

A second investigation by Arthern et al. [1] suggested an anisotropic effect of a snow penetration. Indeed, contrary to [6], their methodology allows distinguishing the subsurfaceecho part due to a snow extinction. They conclude that this anisotropic effect is controlled by the direction of the antenna polarization relative to a buried, wind-induced anisotropy in the structure of the snowpack.

In order to proceed, we investigated this effect on the S-band of the Envisat altimeter. The frequency of this band is smaller by a factor 4.5 than the main $\mathrm{Ku}$-band channel, so that the volume extinction is significantly weaker. The loss by scattering for S-band is almost negligible [7], and the loss by absorption is 4.5 times less than for $\mathrm{Ku}$-band. The anisotropic effect for $\mathrm{S}$-band is found to be much weaker and noisier than for the $\mathrm{Ku}$ band. In Fig. 1(c) and (d), large patterns as observed in Fig. 1(a) and (b) are not detected, except near the coast, especially in the Wilkes Land (from $80^{\circ}$ to $150^{\circ}$ ). Moreover, no correlation between the crossover differences in Ku-band with differences between both bands is found, suggesting that the amplitude of the anisotropic effect is not correlated with the amplitude of the subsurface echo. Clearly, we can only conclude that S-band is less sensitive than Ku-band to the effect. With our actual knowledge on the behavior of electromagnetic waves within the snowpack, these statements somewhat support the hypothesis of the anisotropy in the snowpack characteristics, namely the hypothesis of Arthern et al. [1]. Nevertheless, due to our poor knowledge of the behavior of the electromagnetic waves with respect to the surface roughness for different wave frequencies, we cannot exclude the hypothesis in [6]. Without an electromagnetic model of the polarized echo from the surface to the snowpack, we cannot provide a conclusive evidence.

Finally, note that both studies agree with the fact that this anisotropic effect is due to the sensitivity of the subsurface 
echo with respect to the surface or the snowpack wind-induced anisotropy, so that the effect is modulated by the direction of the antenna polarization relative to the wind direction. The only divergence between the two studies lies in the physical cause.

In this paper, "anisotropy" refers to any sensitivity of the backscattered power or retrieved elevation to the azimuthal orientation of the polarization vector from whatever cause. "Anisotropy direction" refers to the azimuthal orientation of the polarization vector that maximizes the backscattered power.

\section{B. Modeling This Effect}

In any case, the total power Pt received by the altimeter is more or less sensitive to the volume power Pv, depending on the orientation $\Theta$ between the antenna polarization direction and the direction of the wind-induced features and can thus be written as

$$
\operatorname{Pt}=\operatorname{Pref}+\alpha \operatorname{Pv} f(\Theta)
$$

so that, at the crossover point, the difference between the received power $\mathrm{dP}$ is given by

$$
\mathrm{dP}=\alpha \operatorname{Pv}\left[f\left(\Theta_{\mathrm{a}}\right)-f\left(\Theta_{\mathrm{d}}\right)\right]
$$

where $\Theta_{\mathrm{a}}$ and $\Theta_{\mathrm{d}}$ are the angles between the sastrugi-field direction $\xi$ and the polarization directions of the ascending and descending tracks, respectively, where $\alpha$ is the degree of polarization, already defined by Legrésy et al. [6]. It expresses the local amplitude of the surface or snowpack anisotropy and is comprised between zero (no anisotropic effect, smooth surface, and thus no crossover effect) and one (maximum anisotropic and crossover effects).

The function $f(\Theta)$ expresses the modulation due to the orientation. It has been demonstrated in [6] that a cosine function allows to accurately reproduce the observation with the help of the scatterometer data. Note that the relation with the geometrical configuration is independent of the physical cause, so that this modeling can be used.

\section{Occurrence of This Effect at Crossover Points}

The angle $\Theta$ can be deduced from the satellite direction $\beta$, the angle between the satellite direction and the polarization direction $p\left(120^{\circ}\right.$ backward looking for the ERS and Envisat satellites) and the sastrugi-field direction $\xi$ (see Fig. 3 for the geometric configuration), so that

$$
\Theta=\beta+p-\xi
$$

To ensure symmetry, the angle $\Theta$ is given modulo $\pi$, between $-90^{\circ}$ and $90^{\circ}$.

The angle between the meridian and the satellite direction $\beta$ depends on the satellite inclination $\lambda_{\mathrm{M}}$ and the given latitude $\lambda$ and is given by

$$
\beta=90^{\circ}-\lambda_{\mathrm{M}} \cos \left(a \sin \left(\lambda / \lambda_{\mathrm{M}}\right)\right) .
$$

Depending on the track direction, ascending or descending, the angle is either given in the clockwise or counterclockwise direction.

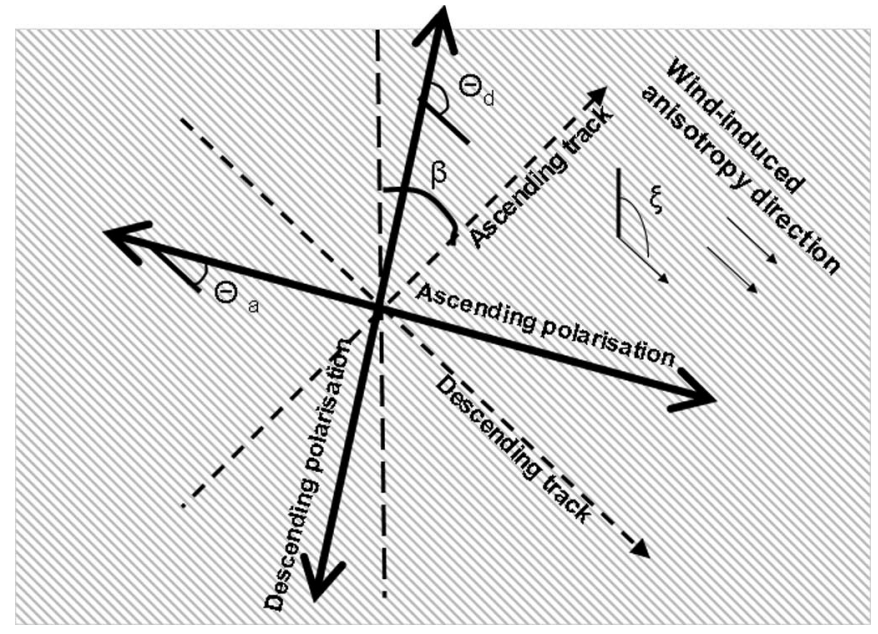

Fig. 3. ERS or Envisat configuration at $70^{\circ} \mathrm{S} . \lambda$ is the angle between the satellite track and the meridian, $\Theta_{\mathrm{a}}$ and $\Theta_{\mathrm{d}}$ are the angles between the antenna polarization and the sastrugi-field orientation for the ascending and descending tracks, respectively.

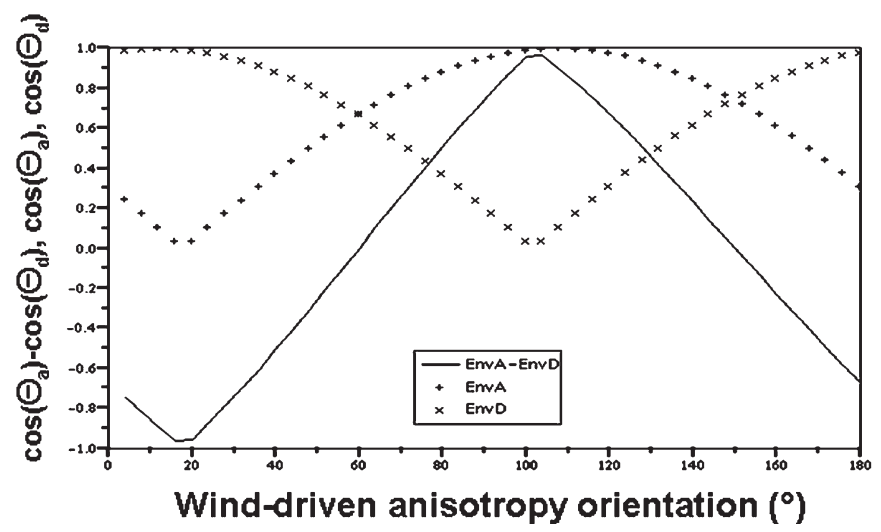

Fig. 4. Induced relative fluctuations in the backscattering-coefficient signal on the altimeter for both tracks and for the difference [see (1) and (2) for the definition]. The exact amplitude depends also on the volume echo and on the degree of the surface anisotropy.

For instance, the ERS and Envisat satellites, whose inclination is $81.6^{\circ}$, cross the meridian at latitude of $70^{\circ}$ with an angle of $48^{\circ}$ for ascending track and $-48^{\circ}$ for a descending track. The angle of the antenna polarization is $120^{\circ}$ backward looking, so that the angle between the antenna polarization direction and the meridian (modulo $\pi$ because of the symmetrical geometry) is $108^{\circ}$ for ascending track and $12^{\circ}$ for the descending one (see Fig. 3 for the geometrical configuration).

As shown in Fig. 4, the power received by the altimeter is modulated as a function of the sastrugi-field direction, so that the difference varies around $\pm \alpha \mathrm{Pv}$ [see (2)]. One can then interpret the pattern displayed in Fig. 1. The backscattering coefficient as well as the whole waveform and the retrieved height value are affected at the $10-\mathrm{km}$ scale due to geometrical variations in the sastrugi-field orientation and the satellite direction. The amplitude at $70^{\circ} \mathrm{S}$ suggests that $\alpha \mathrm{Pv}$ can reach $1.5 \mathrm{~dB}$, and the induced error on the altimetric height is $\pm 1 \mathrm{~m}$.

The induced anisotropy effect slightly decreases with the latitude as the ascending and descending tracks become more and more parallel. At $80^{\circ}$, the amplitude of this effect is reduced by a factor of two with respect to the effect at $70^{\circ} \mathrm{S}$. 


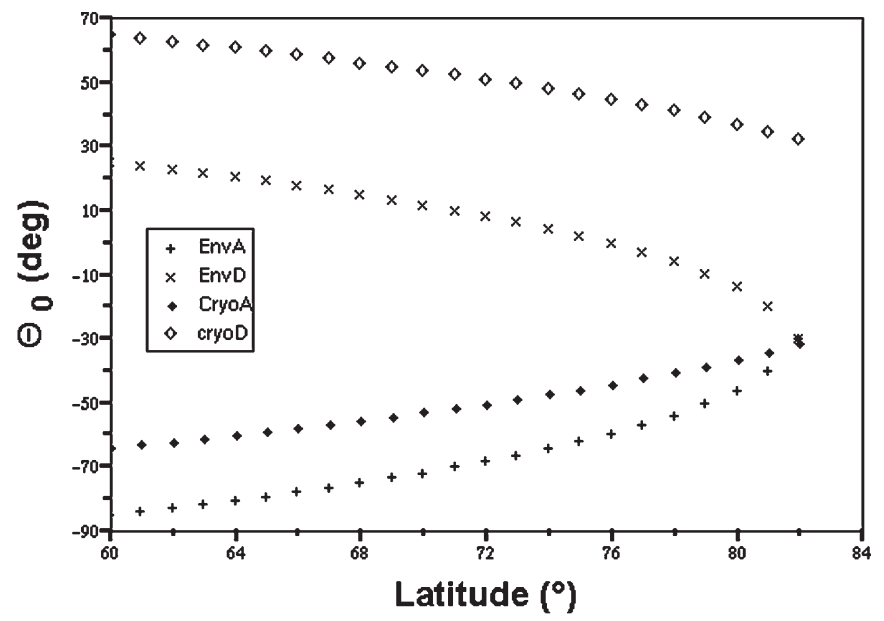

Fig. 5. Evolution of the absolute antenna polarization direction for the ascending and descending tracks of Envisat and CryoSat with respect to the latitude.

\section{Case of Two Different SATEllites}

\section{A. Envisat and Cryosat-2}

The absolute antenna polarization direction depends on the antenna polarization direction in relation to the satellite track and to the direction of the track, i.e., on the local latitude and on the satellite inclination $\lambda_{\mathrm{M}}$ (4). For the CryoSat-2 altimeter, the antenna polarization is perpendicular to the satellite motion, and the inclination of the orbit is $92^{\circ}$. These two differences in comparison to the ERS and Envisat satellites induce a different evolution of the antenna polarization direction with respect to the latitude. Fig. 5 shows the evolution of the absolute antenna polarization direction with respect to the latitude for the four different tracks. This angle tends to close for the ascending tracks of Envisat and CryoSat-2 (the difference is less than $20^{\circ}$ ), but the difference increases between the descending tracks of Cryosat-2 and Envisat and between ascending and descending tracks of CryoSat-2.

The modulation of the signal due to the anisotropy with respect to the anisotropy direction, for the six different combinations of crossover, is displayed in Fig. 6. At a latitude of $70^{\circ} \mathrm{S}$ [Fig. 6(a)], the effect at crossover between the ascending and descending tracks of CryoSat-2 has exactly the same order of magnitude as for Envisat, namely a root mean square (rms) of 0.58 and 0.60 , respectively, for all anisotropy directions. At this latitude, only the crossover difference between the ascending tracks of Envisat and CryoSat-2 is slightly smaller than the other combinations (about $30 \%$ of the Envisat value with an rms of 0.2). All other combinations have their own maxima and minima but with the same amplitude. At a latitude of $80^{\circ} \mathrm{S}$ [Fig. 6(b)], the effect for Envisat crossovers decreases by a factor of two (rms of 0.34), and the effect between the ascending tracks of CryoSat-2 and Envisat decreases by a factor of four to five (rms of 0.11). On the contrary, the effect for CryoSat-2 crossovers (the rms is still 0.58) and for the mixed crossovers between Envisat and the descending CryoSat-2 are still significantly high (rms of 0.6 and 0.48 , respectively).

Furthermore, the anisotropy effect that modifies the altimetric signals will also affect the CryoSat-2 altimeter. Unlike the ERS or Envisat, for which the effect decreases with the
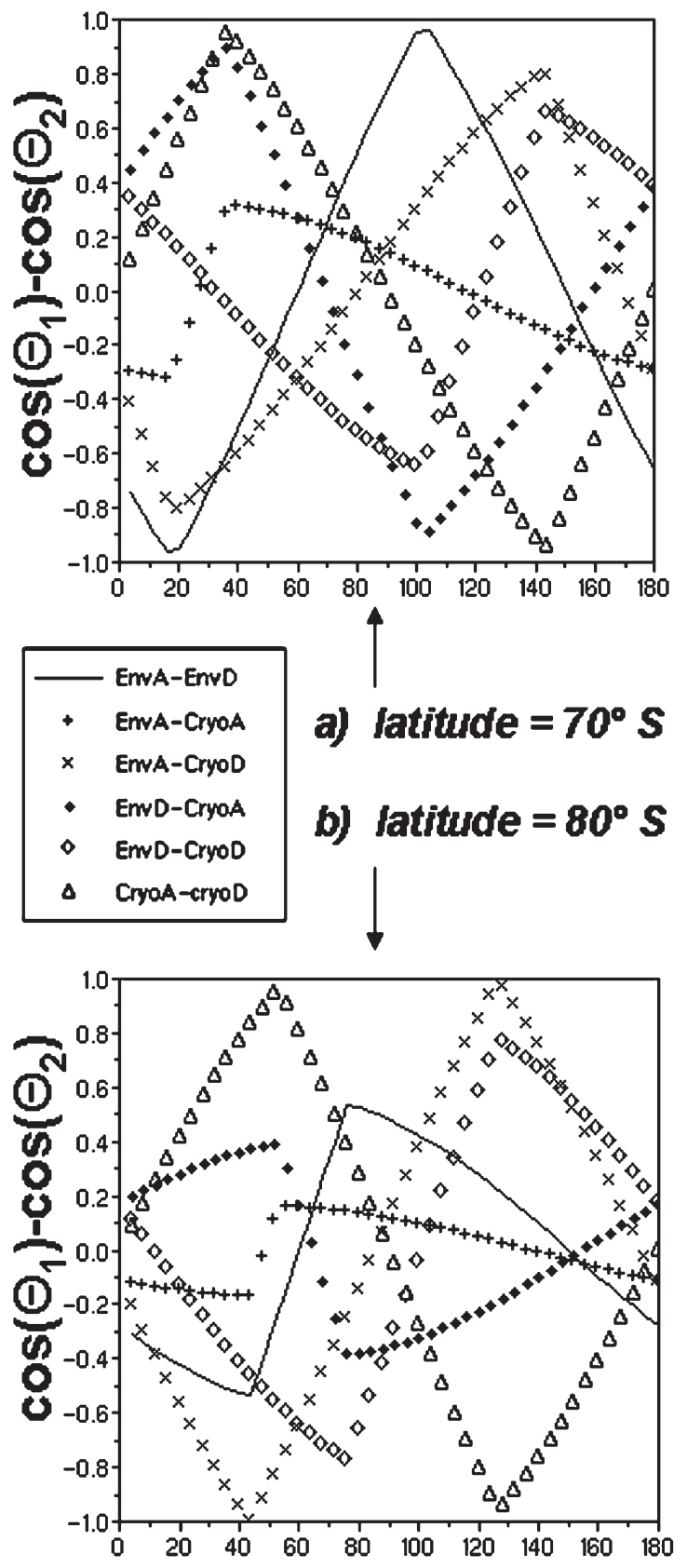

\section{Sastrugi field orientation $\left({ }^{\circ}\right)$}

Fig. 6. Modulation of the anisotropy-induced effect with respect to the anisotropy direction for the six different combinations of crossover. (a) For latitude $70^{\circ}$. (b) For latitude $80^{\circ}$. Note that the effect for Envisat decreases with the latitude, while it is still very significant for CryoSat even at a high latitude.

latitude, the effect for CryoSat-2 will have the same amplitude over the whole continent except near the latitude of $88^{\circ}$. This will make it very difficult to recover the topography with the required precision. Also, the classical crossover analysis will not be possible for CryoSat-2 data processing unless this bias is removed.

There is a significant effect for the four different combinations of crossover between the ascending and descending tracks of both missions. For the backscattering coefficient, one can assume a difference in amplitude of $1.5 \mathrm{~dB}$. In terms of 


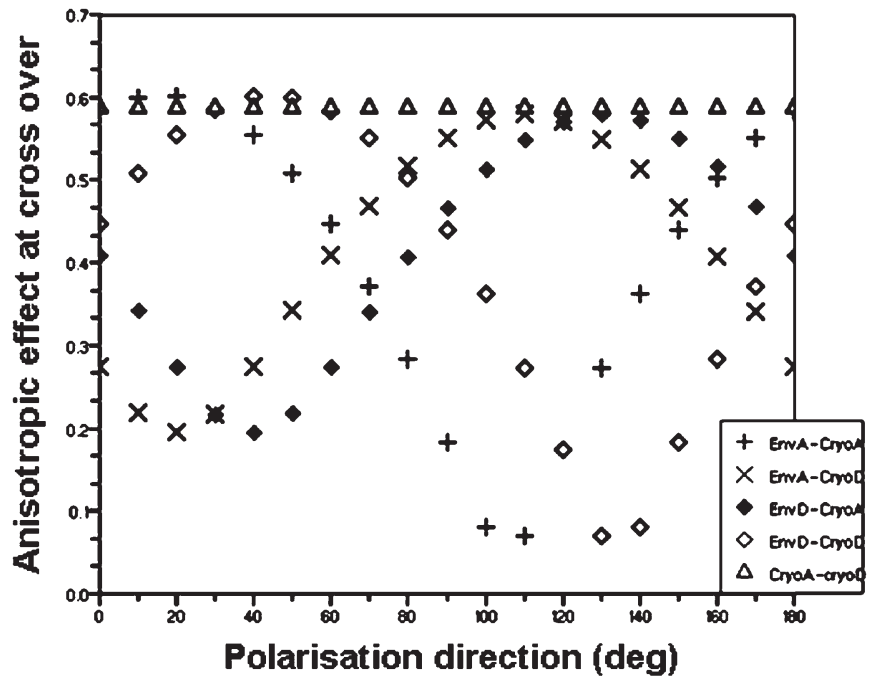

Fig. 7. Root mean square of the anisotropic effect for all latitudes comprised between $70^{\circ}$ and $81.6^{\circ}$ and all anisotropic directions with respect to the CryoSat-2 antenna polarization direction. Note that some polarization directions ensure the possibility to calibrate both missions with respect to one kind of crossover.

elevation, the crossover difference may reach $1 \mathrm{~m}$ over most of the Antarctica ice sheet. This is enough to complicate both comparison and calibration of the two missions.

\section{B. Envisat and a Polar Altimeter}

In this section, we assume that the choice of the polarization antenna for CryoSat-2 or for another polar mission devoted to ice is open. One can wonder what the optimal polarization direction is, in order to minimize the crossover effect. We thus estimate the rms of the effect for each different crossover for all latitudes comprised between $70^{\circ}$ and $81.5^{\circ}$, and for all anisotropic directions (from $0^{\circ}$ to $180^{\circ}$ ) with respect to the polarization direction.

Fig. 7 suggests that the rms induced effect decreases around 0.1 , namely $10 \%$ of the maximum effect for the crossover between both ascending missions and both descending missions if the polarization direction is between $100^{\circ}$ and $110^{\circ}$ and between $130^{\circ}$ and $140^{\circ}$, respectively. If the polarization direction of the new altimeter is around $110^{\circ}$, the rms for both ascending missions decreases near 0.05 for the latitude comprised between $75^{\circ}$ and $81^{\circ}$. On the contrary, if the polarization direction is around $130^{\circ}$, the rms for both descending missions decreases near 0.05 for the latitude comprised between $70^{\circ}$ and $75^{\circ}$.

Choosing appropriately the polarization direction from the above results could minimize the calibration errors when both missions are intercalibrated, and minimize the residual error when time series are constructed from two different satellites.

\section{Use of Both Altimetric Missions to AcQuire INFORMATION ABOUT THE ROUGHNESS ANISOTROPY SIGNAL}

\section{A. Space and Time Configuration of the Problem}

One may wonder whether such a difference between the simultaneous observations for different crossover configurations

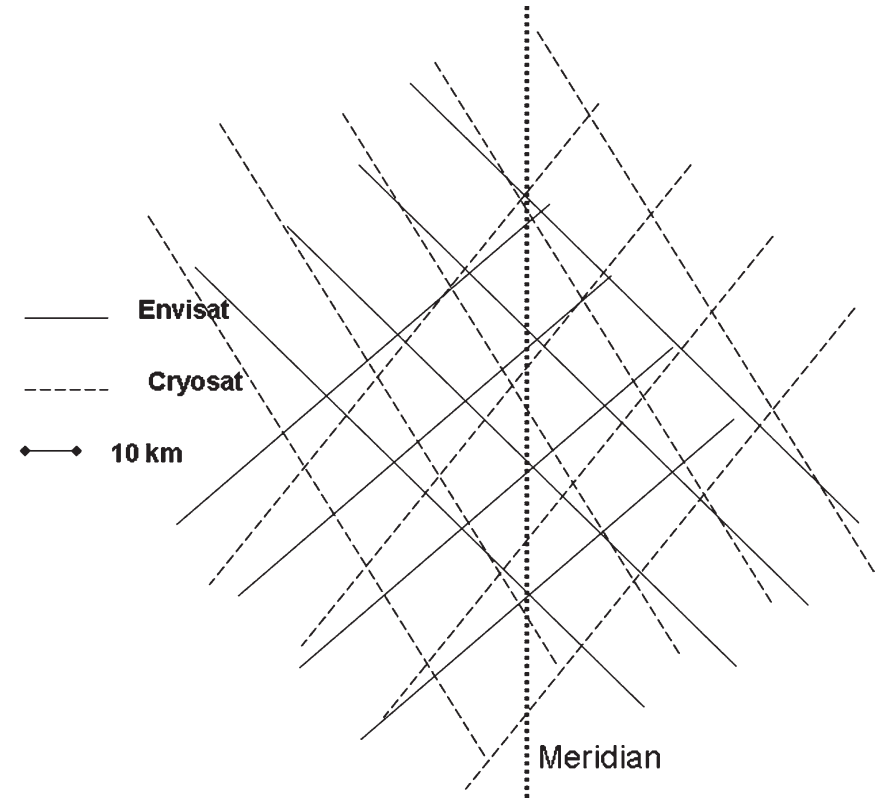

Fig. 8. One 35-day cycle of Envisat is superimposed to one 30-day subcycle of CryoSat, at a latitude of $70^{\circ} \mathrm{S}$. Note the link between the size of the area-i.e., the spatial resolution - and the number of crossover points.

may be used to acquire the information about this anisotropic effect or direction with the help of two different altimetric missions. In other words, can we recover both the anisotropy direction and the volume echo modulation? Both parameters are important for glaciological studies, and their knowledge could be used to correct the different altimetric observations for this complex effect.

Two independent crossover points are theoretically enough to solve the unknown amplitude effect ( $\alpha \mathrm{Pv}$ ) and the anisotropy direction $\xi$ (2). In practice, due to the presence of noise in the measurements, the more independent crossover points we use, the better the precision, which remains only to optimize the space and the time sampling with the needed precision for retrieving the parameters, as shown in Fig. 8. The Envisat repeat cycle is 35 days while it is 369 days for CryoSat- 2 with a subcycle of 30 days. If one wishes to invert these data with a 30-day resolution in order to look at the intraannual and seasonal fluctuations, there are on average two different crossover points for a radius of $10 \mathrm{~km}$, four for a radius of $15 \mathrm{~km}$, and six for a radius of $30 \mathrm{~km}$, at a latitude of $70^{\circ} \mathrm{S}$. This spatial resolution is enhanced with the latitude (improved by a factor of two at a latitude of $80^{\circ}$ ) and, above all, may be enhanced with the temporal resolution. Due to the high density of the CryoSat-2 ground tracks, the spatial resolution can be improved by a factor of ten if the temporal resolution is degraded by a factor of ten (leading then to a mean annual estimation).

\section{B. Opportunity for Parameters Retrieval}

We have simulated several backscattering-coefficient observations at the crossover points corresponding to (2) for different input data. The anisotropy direction varies within the range from $0^{\circ}$ to $180^{\circ}$, and the term $\alpha \mathrm{Pv}$ is always assumed to be one because of its linear impact. For a given anisotropy direction, we estimated for each track the angle $\Theta$ between the antenna polarization and the roughness anisotropy direction. 

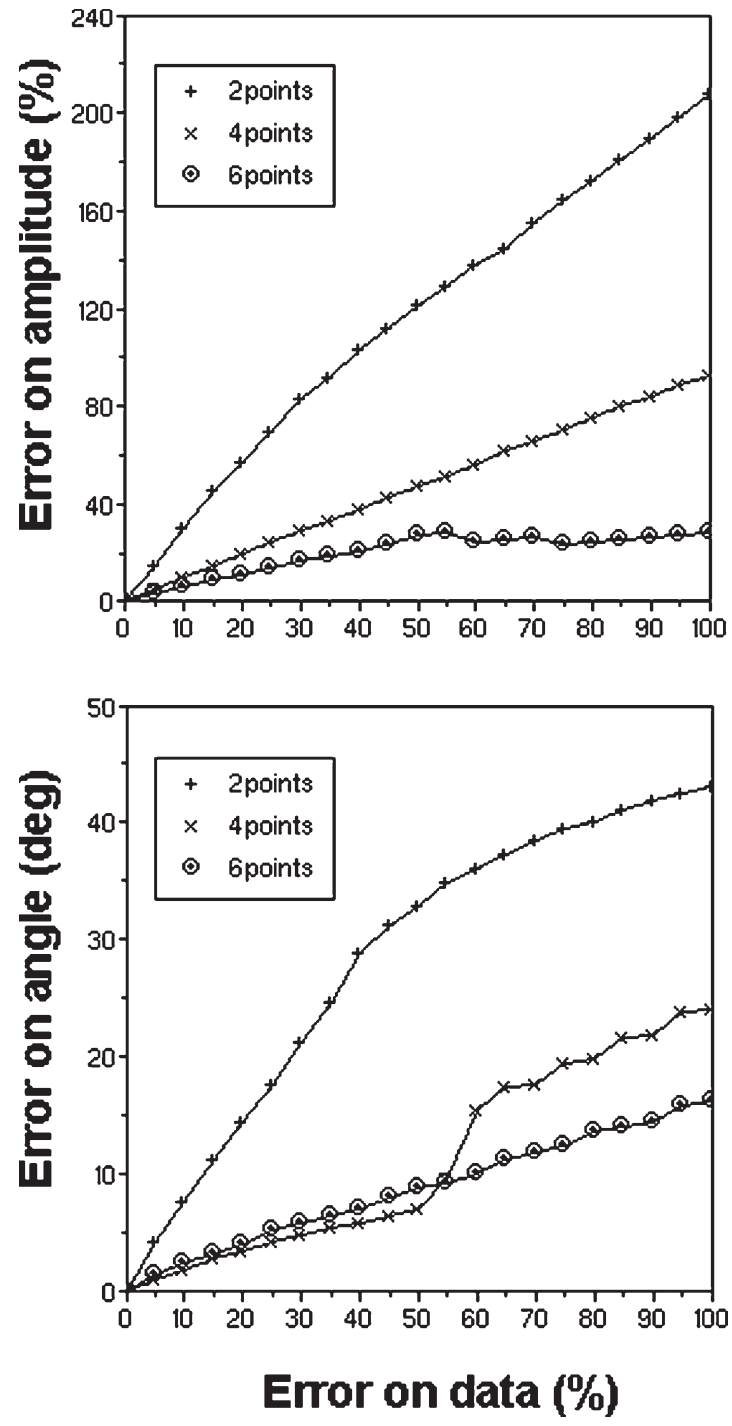

Fig. 9. Simulated inversion of the crossover observations using two, four, or six crossover points with respect to the initial noise on the backscatter. (a) Restitution of the amplitude of the effect expressed in percent. (b) Restitution of the roughness anisotropy direction expressed in degrees.

We assume that within the small selected area, in which the inversion is performed, the noise $N_{i}$ is constant over each track. The noise is assumed to be proportional to the modulation due to roughness anisotropy, meaning that for each track $i$

$$
P(\operatorname{track} i)=\operatorname{Pref}+\alpha \operatorname{Pv} \cos \left(\Theta_{i}\right)+\alpha \operatorname{Pv} \cos \left(\Theta_{i}\right) N_{i} \text {. }
$$

$N_{i}$ is assumed to have a Gaussian distribution, and its rms varies from 0 to 1 , i.e., from 0 to $100 \%$ of the studied signal. It includes the backscattering noise and the temporal changes in the snowpack properties. We simulate the signal for the four tracks and the six crossover points.

The inversion scheme is a classic one. The angle is estimated by a least square analysis and the intensity by a linear fit. To invert, we used two, four, or six independent crossover points. The results with respect to the simulated noise are shown in Fig. 9.

Depending on the noise, taking into account, only two crossover points lead to a poor restitution of the amplitude whose error is twice the initial one in the data. As soon as the initial error becomes greater than $30 \%-40 \%$, the angle is retrieved with an error of $30^{\circ}$. On the contrary, when six crossover points are used, the precision of the retrieved parameter is acceptable: around a few percent for the amplitude and a few degrees for the angle, even for an initial large noise. Note that the noise may be smaller for CryoSat-2 than for Envisat.

Depending on the altimetric noise, the parameters responsible for the anisotropy error may then be mapped precisely. Whenever the sastrugi fields are assumed to be stationary, the mean annual roughness direction may be estimated with a precision better than a few degrees for a radius of $3 \mathrm{~km}$.

The relation between the backscatter modulation and the leading edge (or height) modulation is nearly linear with a correlation coefficient of 0.85 . However, the exact relation slightly depends on the geography, since it is tied to the ratio between the surface and the subsurface echoes. This relation may be locally improved by comparing changes in the backscattering coefficient and altimetric height for six different crossover points, with six different angle samplings. Further study must be performed in order to correctly retrieve and correct for the effect on the altimetric height.

\section{CONCLUSION}

In this paper, we propose a conceptual study on the anisotropy effect on the radar altimetry. The dependence of the altimetric observation on the anisotropy is revealed by its effect at the crossover points. This effect, which is mapped for ERS and Envisat data, has been found to affect the backscattering coefficient by up to $1.5 \mathrm{~dB}$ and elevation by about $1 \mathrm{~m}$. This induced error is the most complex and least understood error to be found in the altimetric data, and it complicates the comparison of different satellites, for instance between the linearly polarized ERS and Envisat altimeters and the previous circularly polarized Seasat and Geosat altimeters. This effect will of course also affect any new altimetric missions with a different orbital or antenna-polarization characteristics, as for example the CryoSat altimeter. The satellite was lost due to a launch failure, but the ESA is rebuilding a New CryoSat-2 satellite to be launched in 2009, and it is expected that new altimetric missions with a polar orbit will fly over the Antarctic ice sheet. In the case of CryoSat-2, due to the polar orbit, the effect will be large over more than $80 \%$ of the Antarctic ice sheet and over the whole of Greenland. It will greatly complicate the classical crossover analysis. Moreover, the effect at crossover between one Envisat track (or an Envisat follow on) and one CryoSat-2 track will also make the comparison difficult and likewise for intercalibration between the two altimetric missions. If ever the polarization direction can be modified from the planned one $\left(90^{\circ}\right)$, we show that an antenna polarization direction for CryoSat- 2 around $110^{\circ}$ or $130^{\circ}$ backward looking minimizes this anisotropic effect.

However, we show that when two different altimeters with different orbit and antenna polarization directions fly together, such as Envisat and CryoSat-2 in the near future, the mixed crossover points give some information on the effect and may help us retrieve both the anisotropy direction and the volume modulation. It then will be an opportunity to better describe this complex and poorly known error. Moreover, we have shown that the space and the time resolution is quite acceptable. The 
mean monthly values of the two unknowns could be estimated with a spatial resolution of around $10 \mathrm{~km}$, while the mean annual values could be estimated with a spatial resolution of less than a few kilometers.

This would enable us to improve the corrections of the altimetric observations, namely the height, waveform-shape parameters, and backscattering-coefficient values and then to improve the height restitution and interpretation of the timeseries data from the altimetric measurements. It would also enable both a seasonal study and a very precise description of the annual roughness and subsurface characteristics. It would offer a new opportunity for improving our understanding on the wind erosion and deposition associated with the katabatic wind and small-scale surface features. Note that the roughness anisotropy estimation is complementary to the one measured by a scatterometer. The scatterometer is a slant-looking radar while the altimeter is a nadir-looking radar, which means that their respective perceptions of the surface roughness are probably not the same. However, both estimations would enable us to address questions regarding the links between the firn layer characteristics and wind-induced structures. However, further studies are needed on the exact physical cause of this effect in order to correctly develop the direct model. We show that the effect is higher for the Ku-band than for the S-band, so that it can point out an origin in subdecimeter scales rather than larger sastrugi or dunes, for which the geometric optics limit applies.

Finally, the anisotropy effect on the radar altimetry described here might also be used elsewhere with other altimeters. For instance, the dual-frequency Topex altimeter is also linearly polarized, but the direction of polarization for the $\mathrm{Ku}$-band and for C-band are perpendicular. This probably has an effect when the difference between both frequencies is used to deduce the surface properties over continental areas (see [9]). Careful analysis should then be performed over surfaces with any apparent roughness anisotropy.

\section{ACKNOWLEDGMENT}

The authors would like to thank F. Blarel and P. Lacroix from Legos, C. Prigent and F. Papa from LERMA at Paris, and the anonymous reviewers for their useful comments.

\section{REFERENCES}

[1] R. J. Arthern, D. J. Wingham, and A. L. Ridout, "Controls on ERS altimeter measurements over ice sheets: Footprint-scale topography, backscatter fluctuations, and the dependence of microwave penetration depth on satellite orientation," J. Geophys. Res., vol. 106, no. D24, pp. 22471-33484, 2001.

[2] A. W. Bingham and M. R. Drinkwater, "Recent changes in the microwave scattering properties of the Antarctic ice sheet," IEEE Trans. Geosci. Remote Sens., vol. 38, no. 4, pp. 1810-1820, Jul. 2000.

[3] D. H. Bromwich, T. R. Parish, and C. A. Zorman, "The confluence zone of the intense katabatic winds at Terra Nova Bay, Antarctica, as derived from airborne sastrugi surveys and mesoscale numerical modelling," J. Geophys. Res., vol. 95, no. D5, pp. 5495-5509, 1990.
[4] M. Frezzotti, S. Gandolfi, and S. Urbini, "Snow megadune in Antarctica: Sedimentary structure and genesis," J. Geophys. Res., vol. 107, no. D18: 4344, pp. 1-12, 2002.

[5] M. Ledroit, F. Remy, and J. F. Minster, "Observation of the Antarctica ice sheet by seasat scatterometer: Relation to Katabatic wind intensity and direction," J. Glaciol., vol. 39, no. 132, pp. 385-396, 1993.

[6] B. Legrésy, F. Remy, and P. Schaffer, "Difference in ERS altimeter measurements between ascending and descending tracks caused by wind induced features over ice sheets," Geophys. Res. Lett., vol. 26, no. 15, pp. 2231-2234, Aug. 1999.

[7] B. Legrésy, F. Rémy, F. Papa, M. Van den Bossche, and O. Z. Zanife, "Validation and tuning of the ICE2 retracking algorithm," Remote Sens. Environ., vol. 95, pp. 150-163, 2005.

[8] D. G. Long and M. R. Drinkwater, "Azimuth variation in microwave scatterometer and radiometer data over Antarctica," IEEE Trans. Geosci. Remote Sens., vol. 38, no. 4, pp. 1857-1870, Jul. 2000.

[9] F. Papa, B. Legrésy, and F. Rémy, "Analysis of the Topex/Poseïdon altimeter over continental area at the global scale," Remote Sens. Environ., vol. 87, pp. 136-147, 2003.

[10] T. R. Parish, "The surface wind field over the Antarctic ice sheets," Nature, vol. 328, no. 6125, pp. 51-54, 1987.

[11] F. Remy, M. Ledroit, and J. F. Minster, "Katabatic wind intensity and direction over Antarctica derived from satellite scatterometer data," Geophys. Res. Lett., vol. 19, no. 10, pp. 1021-1024, May 1992.

[12] D. J. Wingham, A. J. Ridout, R. Scharroo, R. J. Arthern, and C. K. Shum, "Antarctic elevation change from 1992 to 1996," Science, vol. 282, no. 5388, pp. 456-458, Oct. 1998.

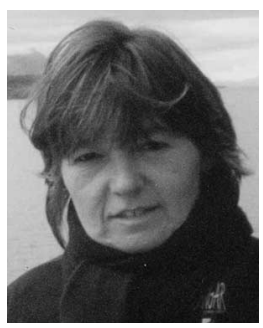

Frédérique Rémy received the Ph.D. degree in 1989.

Since 1989, she has been with the Centre National de la Recherche Scientifique, Toulouse. She is currently the Head of the glaciology team of the Laboratoire d'Etudes en Géophysique et Océanographie Spatiales (LEGOS), Toulouse. She is a specialist of remote sensing of the polar ice sheets.

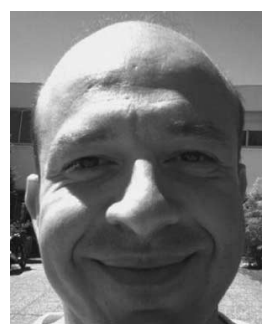

Benoît Legrésy received the Ph.D. degree in 1998.

Since 2000, he has been with the glaciology team at the Laboratoire d'Etudes en Géophysique et Océanographie Spatiales (LEGOS), Centre National de la Recherche Scientifique, Toulouse. He is a specialist of remote sensing of polar ice sheets.

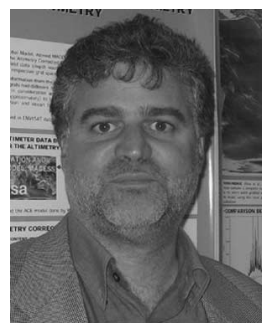

Jérôme Benveniste received the Ph.D. degree in 1989.

Since 1992, he has been with the European Space Agency, Rome, Italy, where he was in charge of the ERS-1 radar altimeter and the Lead of the ERS-2 commissioning calibration and validation working group. He was appointed as Coordinator of the CryoSat radar altimeter ocean cross calibration and validation team prior to the launch failure. 